\title{
Age and gender related variations in human EEG signals
}

\section{H.B.A.C. Pradeep and R.G.N. Meegama*}

\author{
Apple Research and Development Centre, \\ Department of Computer Science, \\ Faculty of Applied Sciences, \\ University of Sri Jayewardenepura, \\ Nugegoda, Sri Lanka \\ Email: pradeephbac@gmail.com \\ Email: rgn@sci.sjp.ac.lk \\ *Corresponding author
}

\begin{abstract}
This research is aimed at analysing EEG data streams from FP1 and F7 channels that reflect activities of the human brain with respect to age and gender. It is mainly focused on finding significant variations in brainwaves detected at frontal parietal lobes of children and adults in both genders. EEG data gathering, data extraction, data cleaning and data filtering technologies were used to observe variations in brainwave patterns among children and adults especially in regions of the brain associated with logical thinking and linguistics. We used cross correlation functionality to obtain similarity between subject datasets in the sample space. Results indicate increased active brain signal patterns near the frontal parietal lobe of young male subjects than in female subjects.
\end{abstract}

Keywords: EEG; brain waves; brain activity; human behaviour.

Reference to this paper should be made as follows: Pradeep, H.B.A.C. and Meegama, R.G.N. (2020) 'Age and gender related variations in human EEG signals', Int. J. Digital Signals and Smart Systems, Vol. 4, Nos. 1/2/3, pp.87-99.

Biographical notes: H.B.A.C. Pradeep is pursing his MPhil in the Department of Computer Science. He obtained his BSc in Computer Science from University of Sri Jayewardenepura, Sri Lanka. He is currently pursuing an MPhil in Signal Processing. After his graduation, he has worked in the industry for nearly five years in research and development in topics related to machine learning and AI. His current research interest is investigating the human brain and associated neuron activities. Apart from his research activities, he regularly participates in several technology meet-ups, conferences as a speaker and keep sharing his knowledge with university students among many Sri Lankan universities as a Guest Lecturer.

R.G.N. Meegama obtained his BSc in Computer Science from the University of Colombo and MSc in Computer Science from the AIT, Thailand. He received $\mathrm{PhD}$ in Medical Imaging from the NTU, Singapore in 2004. Presently, he is a Professor in Computer Science at the Department of Computer Science, Faculty of Applied Sciences, University of Sri Jayewardenepura, Sri Lanka. He has published many research articles based on image processing, computer graphics, mobile computing and wireless sensor networks in peer reviewed indexed journals and conferences. He represented Sri Lanka as a Young Scientist at the World Economic Forum in Dalian, China in 2009. 
This paper is a revised and expanded version of a paper entitled 'Correlation analysis of age related diversity in human brain waves' presented at the 3rd International Conference on Advances in Computing and Technology, Sri Lanka, 26 July 2018.

\section{Introduction}

The brain is the most important and the most complex human organ that is responsible for all the functions that we do in our routine life. Moreover, the brain consists of millions of neurons that utilise electro-chemical signals to transmit information to other parts of the body. Whenever a neuron triggers an electrical impulse to another neuron, it is transmitted trough a chemical gap, referred to as a synapse, and generates electricity that can be measured by electronic devices. In addition, such brain waves are directly related to our routine activities and behaviours. As a result, numerous experiments have been conducted to analyse EEG pulses to find out the relationship between several human mental states and also to recognise human behaviour and characteristics. In this study, we analyse such brainwaves obtained from individuals within same environmental conditions to uncover hidden relationships in age and gender. The goal of this research was to identify the differences in brainwave patterns between genders as well as among different age groups. We conducted the study in three main steps, namely data gathering, filtering and processing and observation of results.

\section{Recent work}

In van Baal et al. (2001), a study is conducted to examine the stability and change in genetic and environmental influences on EEG coherence from ages 5 to 7-years. This happens during growth spurts of a child during discontinuous development of mental cognition. Dyadic correlation between a stimulated and a non-stimulated functional state of a brain is analysed in Wackermann (2004).

In another work (Radin, 2004), EEG signals in pairs of people are examined to verify whether event related potentials evoked in a particular person's brain are correlated with concurrent responses inside the brain of another distance but isolated person. This study concludes that under certain conditions, the EEG signals of a sensorial isolated person can be correlated with event-related potentials in a distant person's EEG suggesting the presence of a hitherto unknown form of interaction between the subjects.

The correlation of brain activities of two separated human beings is analysed in Wackermann et al. (2003). This experiment involved recording six channels EEG data simultaneously from pairs of separated human subjects in two acoustically and electromagnetically shielded rooms where one person is subjected to visual pattern-reversal stimuli while the other subject relaxed without any stimulation.

In Miraglia et al. (2015), a graph theory approach is used to quantify differences in brain activity between males and females during resting state. Differences in alpha, delta and gamma bands between males and females are observed in the frontal lobe of the left and right hemispheres indicating variations in EEG patterns during the resting state of the 
brain. Although this study involved 130 subjects (59 males and 71 females), it does not reveal any differences in brain wave patterns with respect to age.

Another study compared the ERP characteristics as the field of distribution, an intensity in three evoke potential readings such as the mismatch negativity (MMN), the P300 and the N400 using a high-density electroencephalogram (HD-EEG) (Tsolaki et al., 2015). This study comprised of 27 young adults and 18 elderly, healthy and right-handed subjects who underwent auditory ERP extraction leading to establishing a relationship between ERP and gender groups. However, the experiment did not exhibit any ERP relationships with respect to age.

A fuzzy-integral based method and a linear dynamic system (LDS) are used in Zhu et al. (2015) to compare patterns in 14 subjects with respect to emotional states such as positive, negative and neutral while watching movie clips. Investigating cross-subject and cross-gender emotion classification revealed only gender variations in EEG patterns.

The effect of different sevoflurane concentrations on the incidence of epileptiform EEG activity during induction of anaesthesia in 100 children, between 1 to 7-years old, undergoing a clinical routine is investigated using a statistical approach (Kreuzer et al., 2014). This study, however, do not present results on EEG patterns with respect to gender differences.

\section{Methodology}

\subsection{EEG data capture}

In this stage, data of 22 subjects were gathered from publicly available sources. All the related information had been gathered by investigators from Children's Hospital, Boston (CHB) and the Massachusetts Institute of Technology (MIT) and subsequently published at PhysioNet (Goldberger et al., 2000). It contained information on five males and 17 females whose ages ranged from 6 to 22-years.

All the data was recorded with $256 \mathrm{~Hz}$ within a 16-bit resolution.

During data collection, the electrodes were placed in accordance with the international 10-20 system as shown in Figure 1 which is a widely accepted standard for electrode placement in EEG related studies. In this research, we have used the data obtained from the FP1 and F7 channels because they are located towards the left frontal lobe of the brain that deals with logical and linguistic tasks as depicted in Figure 1.

Our sample dataset (given in Table 1) contained EEG data gathered from four subjects with nine data samples from each. All the data were collected at random time points. From the existing dataset, we selected four special subjects for our study and the data were gathered from two specific channels (FP1 and F7) as shown in Figure 1. The primary objective of this research is to find out whether there is a difference between males and females with respect to EEG signal patterns emitted from the frontal left brain during logical and linguistic tasks.

Because all the data were gathered from a single research project, we can assume that the environmental conditions were identical for all subjects. As all the data were recorded in .edf file format, we used an external tool to visualise the recorded data and subsequently applied a filtering technique to remove noise. 
Table 1 Extracted subject overview

\begin{tabular}{lcccc}
\hline Subject & Age $(y)$ & Gender & Sample size & Total records \\
\hline A & 11 & M & 9 & 2,304 \\
B & 11 & F & 9 & 2,304 \\
C & 22 & M & 9 & 2,304 \\
D & 6 & F & 9 & 2,304 \\
\hline
\end{tabular}

Figure 1 Placement of electrodes according to international 10-20 system

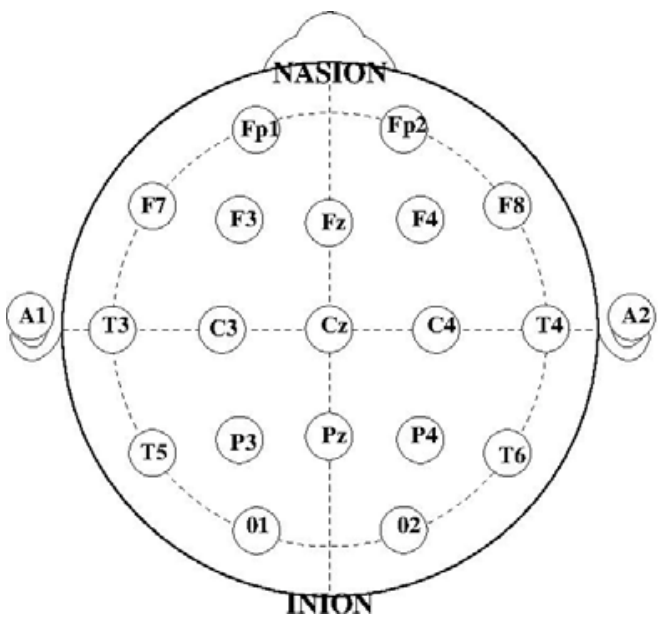

Source: Wackermann (2004) and Selesnick et al. (1998)

As all EEG data used in this project were in .edf file format (Goldberger et al., 2000), data gathering, cleaning and processing involved several additional steps. In the first phase, we applied a filtering process to clean the EEG dataset of young male and female subjects. Thereafter, we converted the .edf files to .csv numerical format and then, using MATLAB, hidden patterns in the cleaned dataset were identified. Finally, we calculated and compared the standard deviation of the wave power in every individual sample with respect to gender.

The data files, each having $81 \mathrm{MB}$ of data, were in human readable .edf format. As such, an EDFBrowser was used to convert .edf file data to a numeric domain. Using this EDFBrowser, we were able to convert .edf data to .csv format containing time domain data of EEG waves as shown in Figure 3(a). Raw EEG data usually contain a certain amount of noise added to the original signal during eye movement, vibrations, etc. As noise signals may lead to erroneous results, a filtering process must be utilised to clean the data. We used the Butterworth filter (Selesnick et al., 1998; Alarcon et al., 2000) to pass frequencies in between the lower and the upper bound frequencies and this equation helps to clear subject dataset for further calculations. The Butterworth filer is designed to extract and reject unwanted frequencies lying outside the range of cut-off frequencies to achieve above task. We used a 6th order band pass filter having cut-off frequencies between $1 \mathrm{~Hz}-8 \mathrm{~Hz}$ to observe signal information. 


\subsection{Extracting sample data from random time ranges}

In this research, we considered EEG data gathered from probes located near the frontal parietal lobe of the brain known as FP1 and F7 in the 10-20 international electrode system (Helmuth et al., 1989). The channel odd numbers were recorded from left side of the brain.

\subsubsection{Cross-correlation analysis}

A correlation function gives the statistical correlation between random variables. In our research, correlation can be calculated between two signal forms (datasets). Correlation functions are a useful indicator of dependencies as a function of distance in time or space. But when we consider EEG signal domain we have to deal with continuous EEG dataset which we cannot define each quantity of data points. Then we must move for cross-correlation to find out similarities between two wave forms. Cross-correlation function is widely used for calculating amount of similarity between two wave forms. In the calculation process one data series will shift value by value to left and right to get value Metrix of co-relation and lag values.

This functionality is also known as a sliding dot product or sliding inner product of two wave forms and commonly searching for a long signal for shorter, known feature.

$$
R(\tau)=\int_{-\infty}^{+\infty} x(t) y(t+\tau) d t
$$

where $x(t)$ and $y(t)$ are functions of time and $\tau$ is the time delay.

Also, we can define cross-correlation as a cross product of the two-time based functions as:

$$
\left(x^{*} y\right)(\tau)=\int_{-\infty}^{+\infty} x^{*}(t) y(t+\tau) d t
$$

where $x^{*}$ denotes the complex conjugate of $x$ and $\tau$ is the displacement, also known as lag, although a positive value of $\tau$ actually means that $y(t+\tau)$ leading to $x(t)$. The cross correlations functions $x(t)$ and $y(t)$ are equivalent to the convolution of $f^{*}(-t)$ and $g(t)$ as follows:

$$
\begin{aligned}
& {[x(t) * y(t)](t)=\left[x^{*}(-t) * y(t)\right](t)} \\
& {[x(t) * y(t)](t)=[x(t) * y(t)](-t)}
\end{aligned}
$$

Analogous to the convolution theorem, the cross-correlation satisfies:

$$
F\{x * y\}=F\{x\}^{*} \cdot F\{y\}
$$

where $F$ denotes the Fourier transform (Bickford et al., 1987) and this property is often exploited for efficient numerical computation of cross-correlations.

The discrete form of the cross correlation can be given as:

$$
(x * y)(\tau)=\sum_{t=-\infty}^{\infty} x(t) y(t-\tau)
$$




\section{Results and discussion}

In the experiments conducted, we have used the signals generated from FP1 and FP7 channels. These nodes are located on the left frontal region of the skull. It has been reported that the left frontal lobe of the brain is responsible for linguistic and logical tasks of the brain and as such, our main aim is to analyse the brain wave potentials in this region associated effects on tasks such as grammatical judgements, lexical decisions, board games and cross word puzzles.

Figure 2 Comparison of brain waves of an 11-year-old male (M) and an 11-year-old female (F) in 256 records, (a) between $2 \mathrm{~s}-3 \mathrm{~s}$ (b) between $11 \mathrm{~s}-12 \mathrm{~s}$ (c) between $21 \mathrm{~s}-22 \mathrm{~s}$

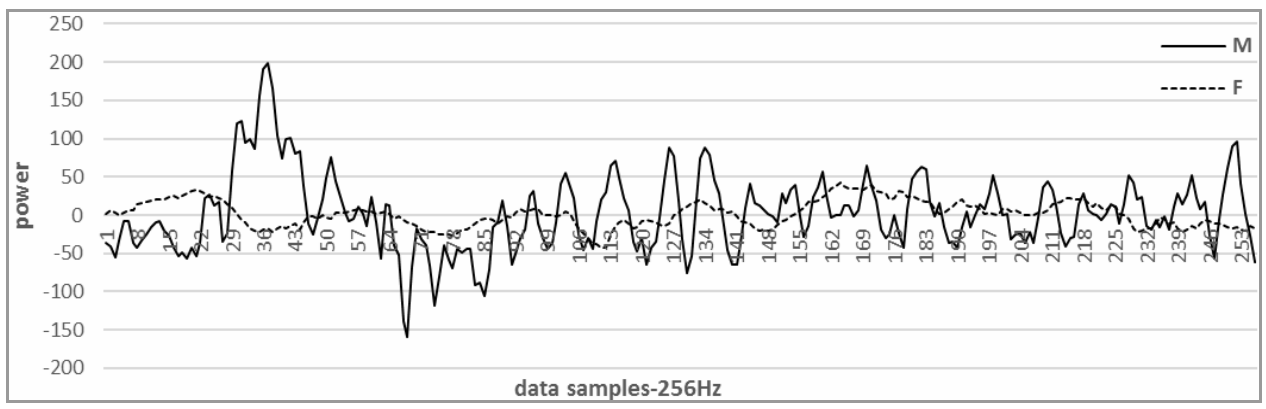

(a)

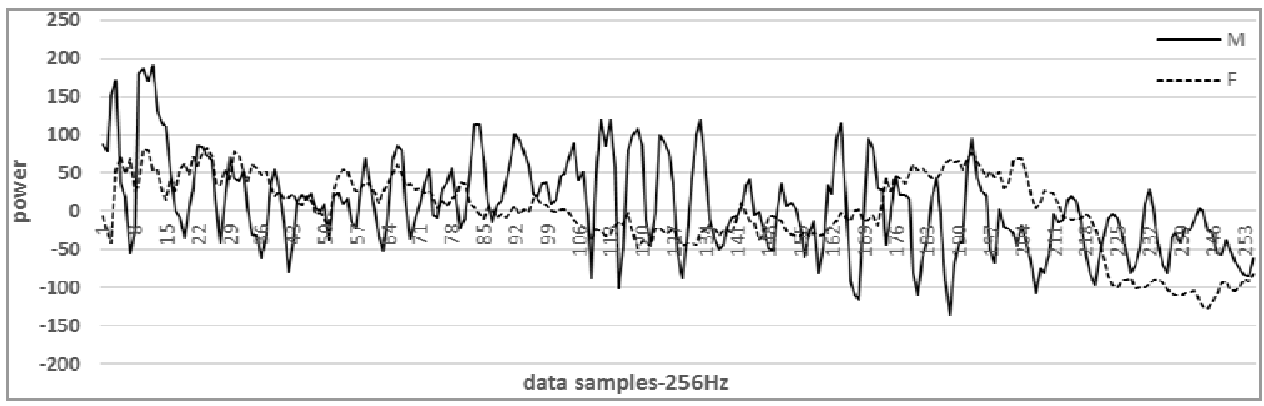

(b)

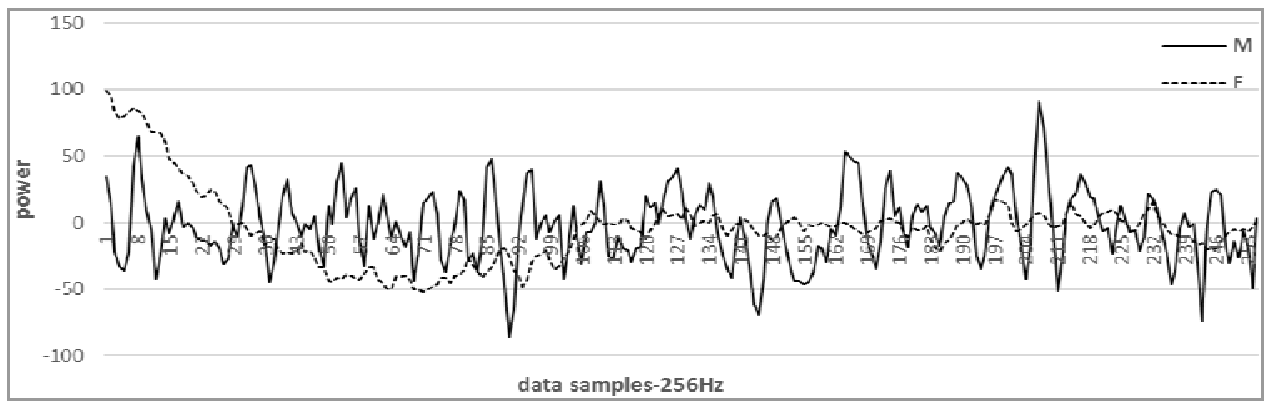

(c) 
Figure 2 depicts the comparison results between a male and female who were both 11 -years old. For this purpose, we added the frequency response in three different time domains only. We ignored the first 256 samples, which were captured during $1 \mathrm{~s}-2 \mathrm{~s}$ time period to compensate for possible errors added to the main brainwave during head movements and early adjustments. Figure 3 gives brain wave patterns of a male and a female subject who were 22 and 6-years, respectively.

Figure 3 Comparison of brain waves of a 22-year-old male (M) and a 6-year-old female (F) in 256 records, (a) between $2 \mathrm{~s}-3 \mathrm{~s}$ (b) between $11 \mathrm{~s}-12 \mathrm{~s}$ (c) between $21 \mathrm{~s}-22 \mathrm{~s}$

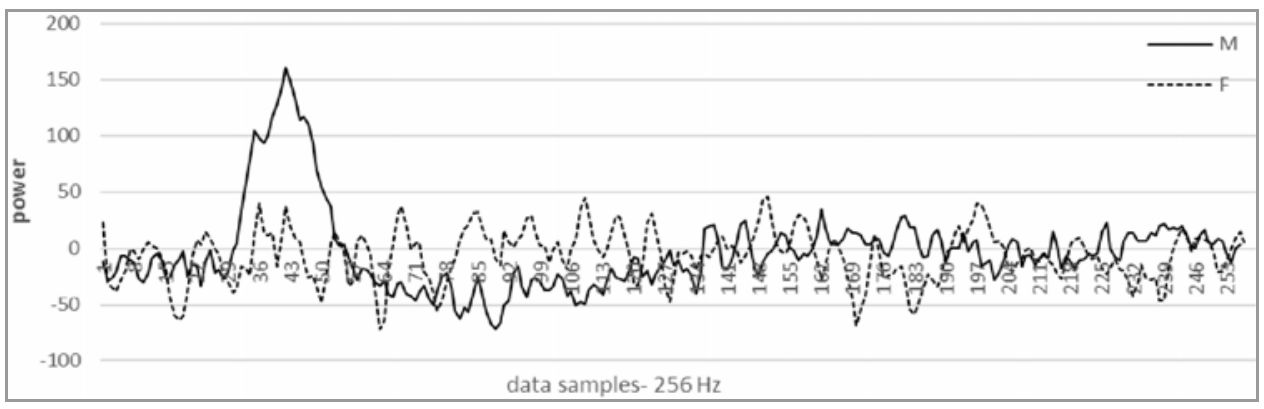

(a)

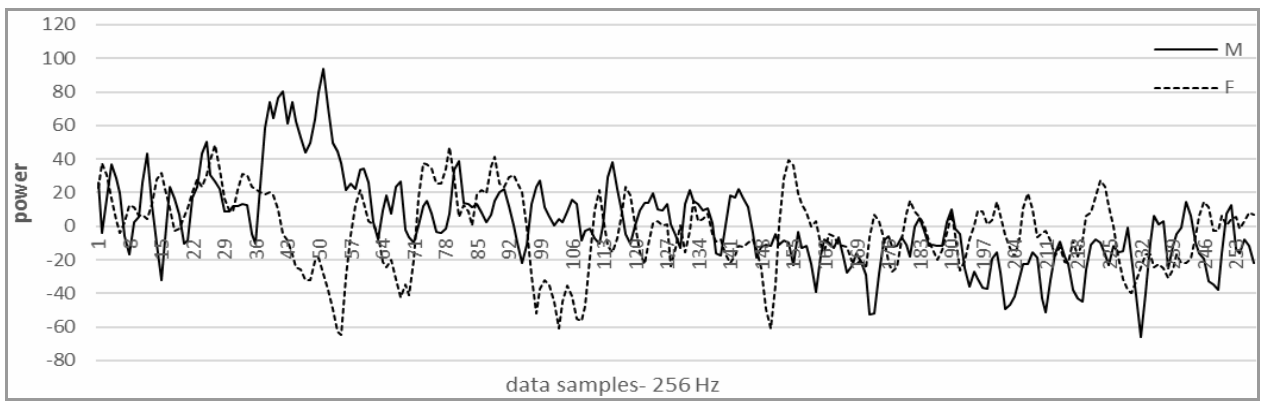

(b)

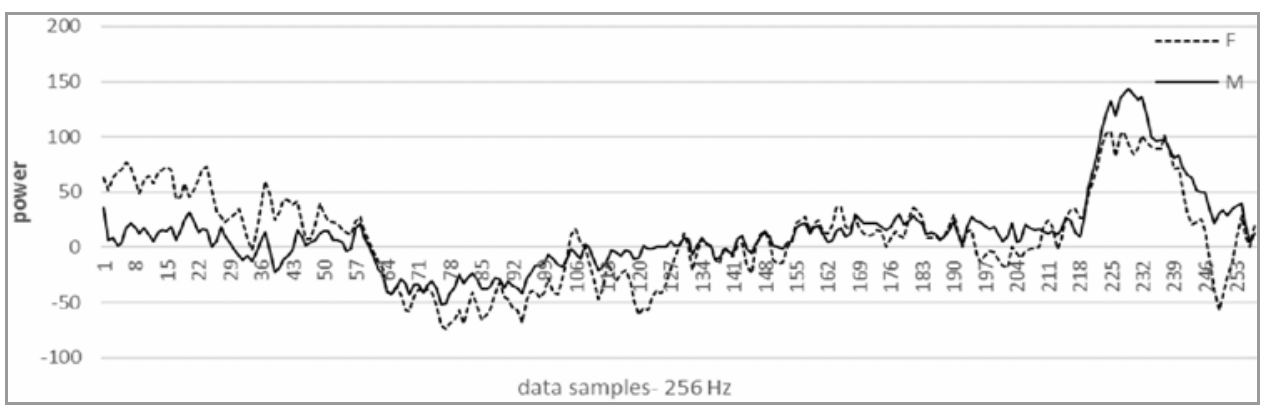

(c) 
As seen in Figure 3(a), we see that in male subjects, brainwave fluctuations are greater than female subjects are in the age 11. In addition, Figure 3(b) demonstrates the signal variations of a male and a female who are 22 and 6-years of age, respectively.

Figure 4 depicts the standard deviation of brainwave potential of male and female subjects whose ages range from 6 to 22-years. High standard deviation of brainwave potential is observed from both male subjects in age groups namely 11-years old age group and 22-years old age group respectively. These observations can be aligned with the fact that within the 11-years old and 22-years old age categories, men show high activity in brain potentials than women in the same age category.

Figure 4 Standard deviations of wave potential: of males (M) and females (F),

(a) both 11-years-old (b) 22-years and 6-years, respectively

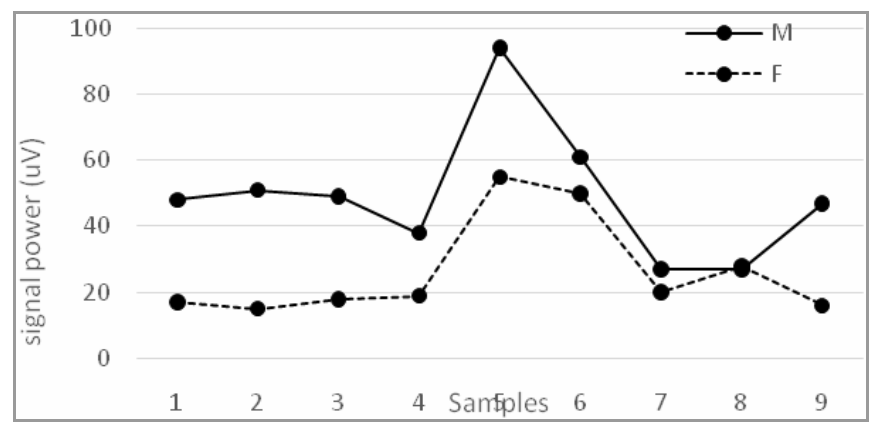

(a)

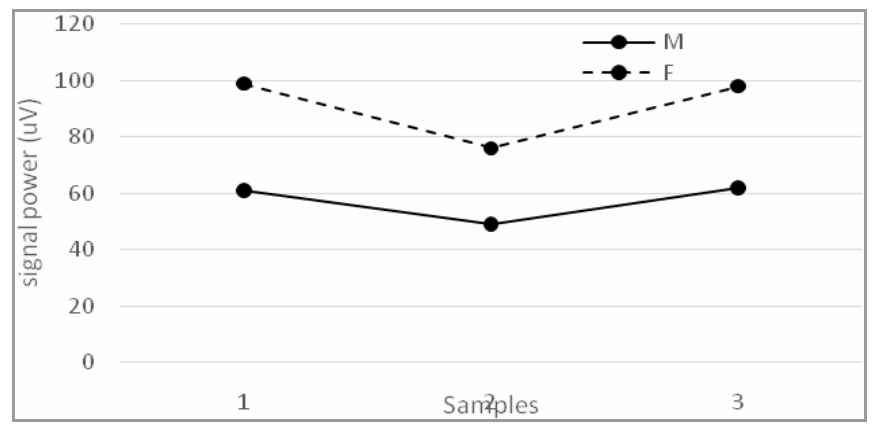

(b)

Finally, after filtering all the data, nine random samples are taken from the dataset as given in Table 2 in which every dataset contains $1 \mathrm{~s}$ of EEG signals consisting of 256 recorded points. The Butterworth filer (Selesnick et al., 1998; Alarcon et al., 2000) is designed to extract and reject unwanted frequencies located outside the range of cut-off frequencies. This filter generates a maximally flat frequency range in between the cut-off frequencies. Moreover, the frequencies generated have no ripples in the pass band and converge towards the zero band. It must be noted that the response of the first order low pass filter rolls off at $-6 \mathrm{~dB}$ per octave, the second order filter at $-12 \mathrm{~dB}$ per octave and the third order filter will start decreasing at $-18 \mathrm{~dB}$ per octave. When we compare the 
Butterworth filter with other linear filters used for signal normalisations, we observed a slight nonlinear phase response in the Butterworth filter than other linear filters and a quick roll-off around the cut-off frequency. In addition, the smoothness of the curve increases with the order (Goldberger et al., 2000).

Figure 5 FP1-F7 EEG sample wave cross correlation comparison, (a) 256 records from age 11 -year male and 11-year female (b) 256 records from age 11-year male and 6-year female (see online version for colours)

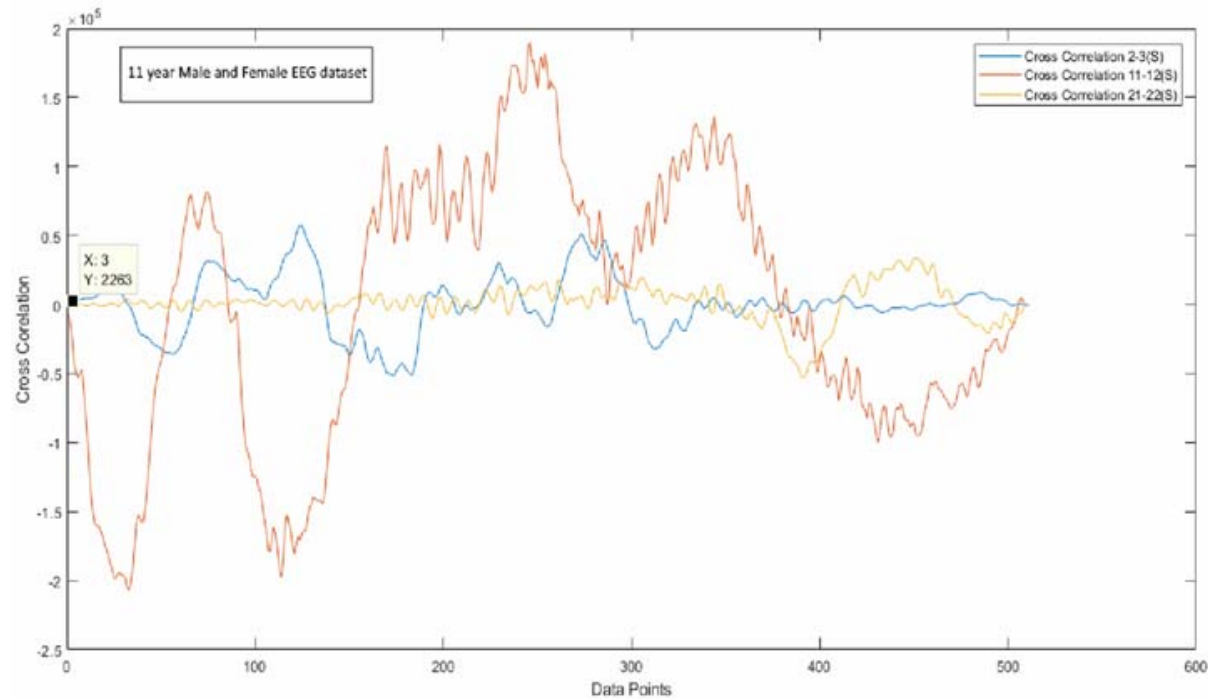

(a)

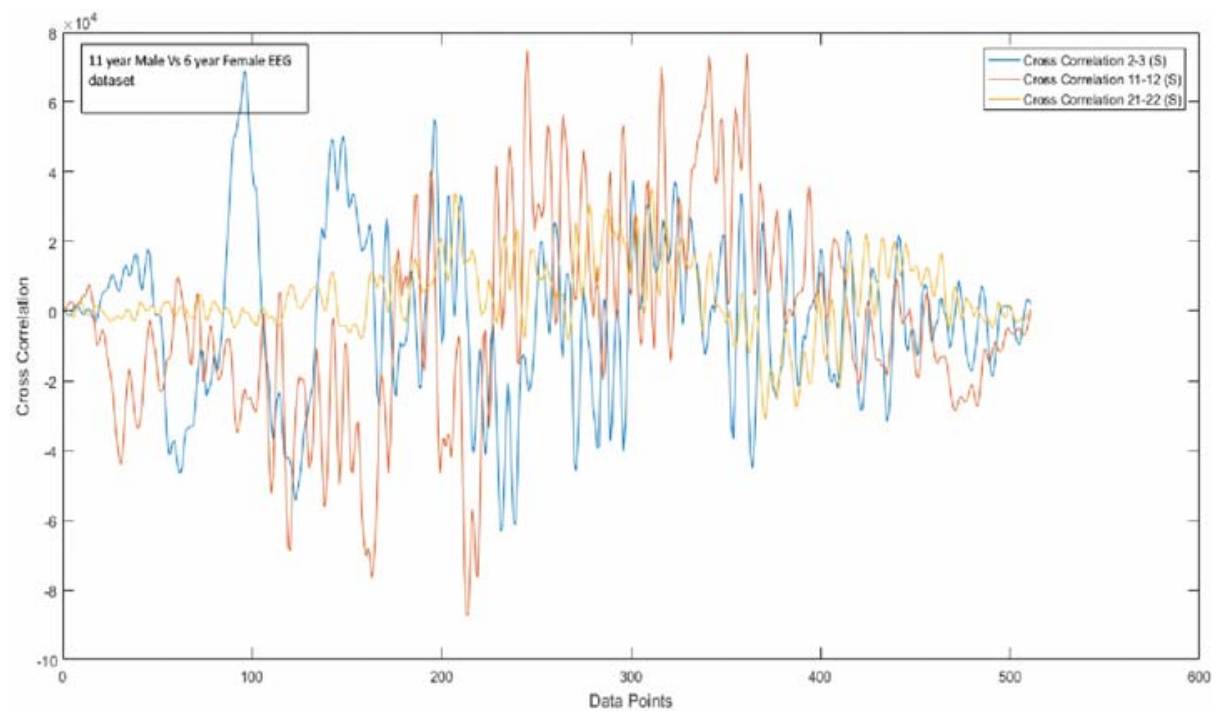

(b) 
Figure 6 FP1-F7 EEG sample wave cross correlation comparison, (a) 256 records from age 11 -year male and 22-year male (b) 256 records from age 22-year male and 6-year female (see online version for colours)

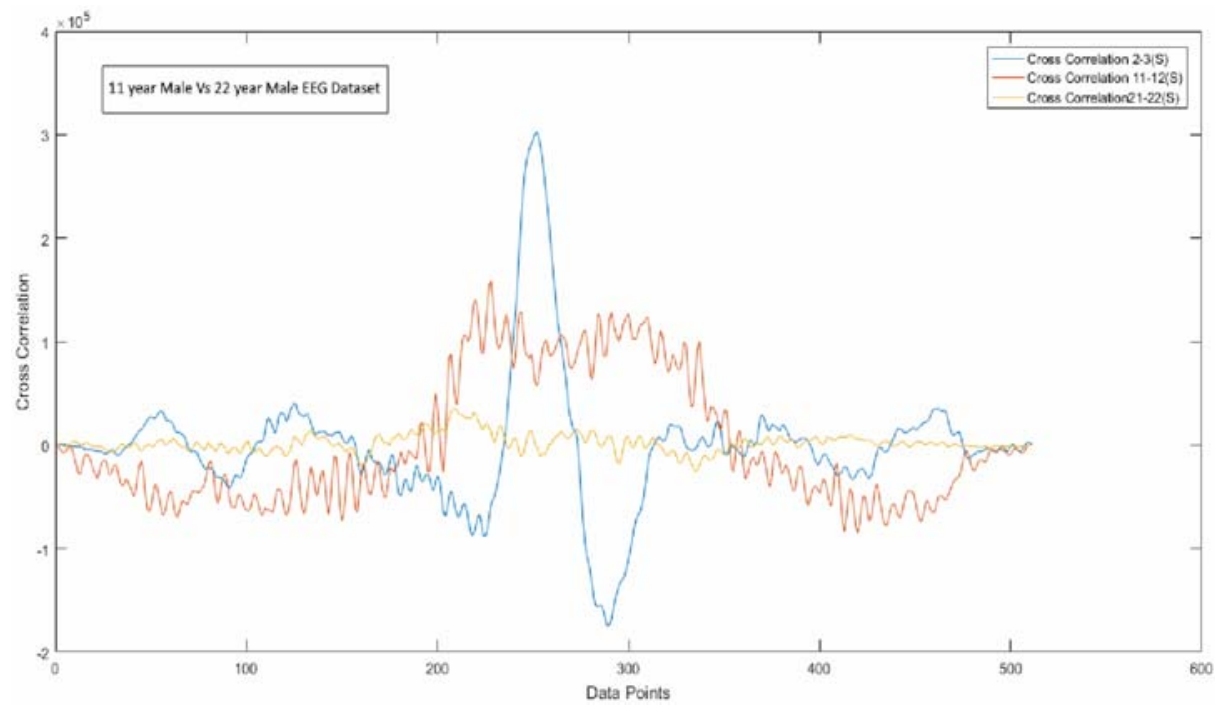

(a)

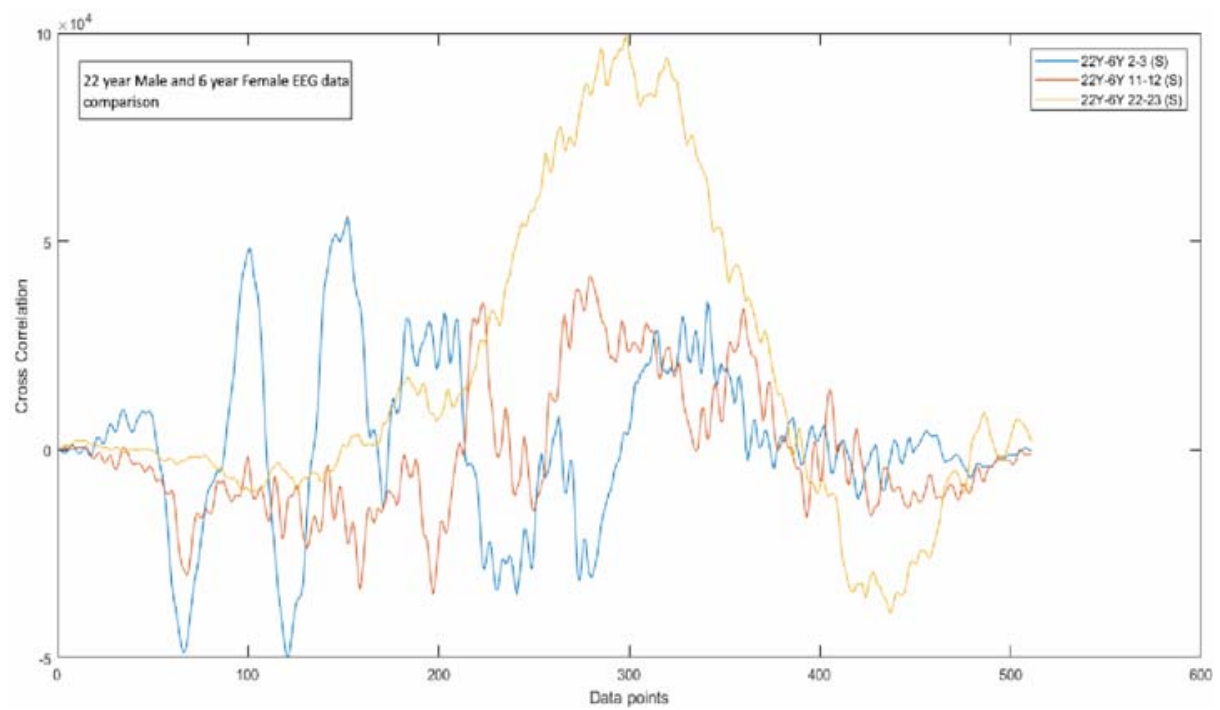

(b)

\subsection{Results of cross-correlation analysis}

For cross-correlation analysis, four different groups of subjects, based on gender and age, are selected as follows in which each group consisting of two subjects. 
a group one: male with female both 11-years

b group two: 11-year male with 6-year female

c group three: 11-year male with 22-year male

d group four: 22-year male with 6-year female.

The specific individuals in the groups are selected to account for different age and gender of the subjects. In other words, groups containing male child and adult, female child and adult, male and female children, female child and male adult are considered for cross-correlation analysis. In the datasets, the collected and cleaned EEG dataset from the FP1-F7 channels relate to left frontal lobe of the brain. We used cross-correlation analysis to find signal similarities in the brain waves between different individuals. In the cross-correlation, it is possible to derive high peaks within the value set against with lag values as seen in Figures 5 and 6.

Figure 7 Cross-correlation normalisation for, (a) age 11-y male and 22-y male at $2 \mathrm{~s}-3 \mathrm{~s}$

(b) age 22-y male vs. 6-y female at $11 \mathrm{~s}-12 \mathrm{~s}$ (c) age $22-\mathrm{y}$ male/female at $21 \mathrm{~s}-22 \mathrm{~s}$ (see online version for colours)

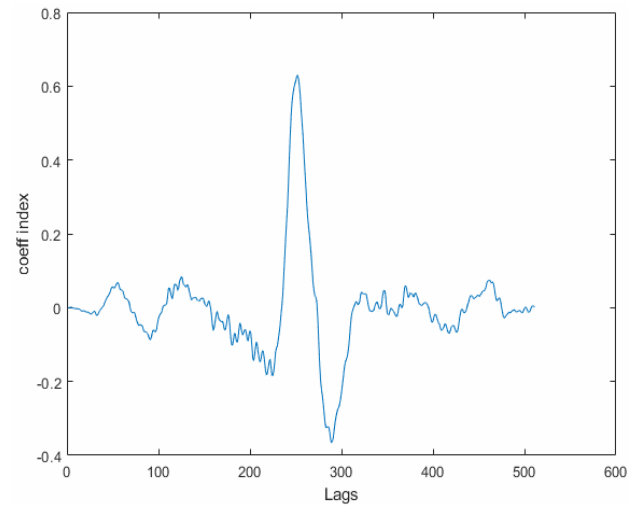

(a)

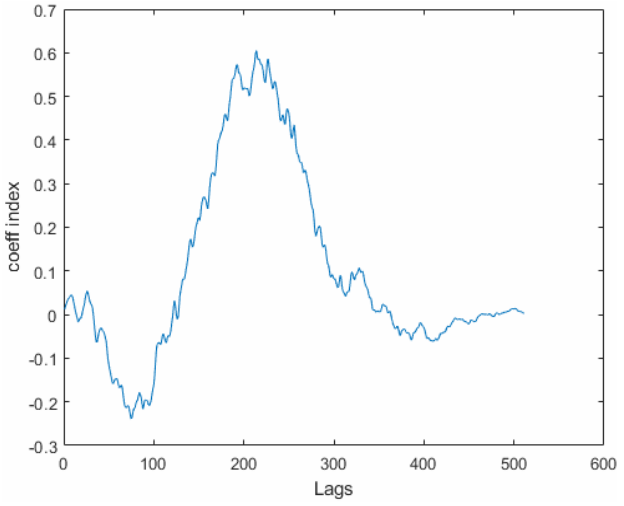

(b)

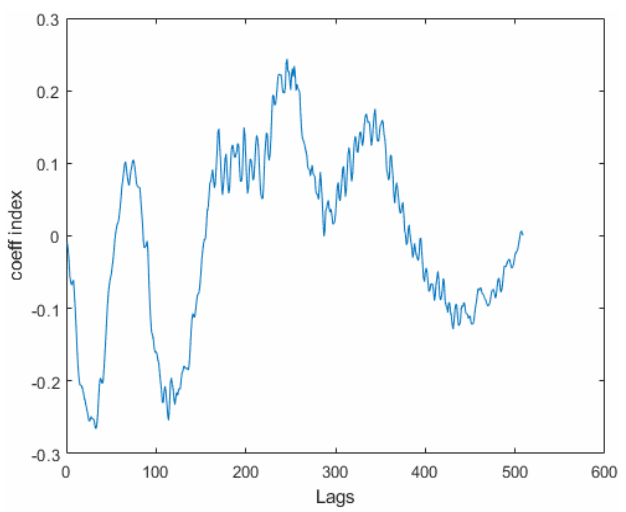

(c) 
Our work is aimed at processing raw EEG data and subsequent filtering mechanism to find out both age and gender effects on brain wave patterns of healthy volunteers. It is worthy to note other similar experiments carried out but limited in scope. For instance, EEG data collected from 117 subjects are used to analyses work-related depression in Tement et al. (2016) using the alpha frequency to match correlation and used regression analysis to identify exact burnout and depression in the subjects. The limitation of this work is that only experiments were done to investigate the effect of only alpha frequencies to detect stages depression among the subjects. Further, a quantitative EEG was used to evaluate characteristics of patients with unmediated schizophrenia during their resting period (Kim et al., 2015) using 180 subjects divided into two categories, namely, schizophrenia patients and healthy subjects. Group differences within the five frequency bands across several brain regions were examined to identify discrimination ability of each frequency band. A proof-of-concept was carried out to evaluate subject-independence based on hand motor imagery with respect to gender using common spatial patterns and linear discriminant analysis (Cantillo-Negrete et al., 2014).

Table 2 Extracted subject overview

\begin{tabular}{lc}
\hline Sample & Peak value (cross-correlation value) \\
\hline 11 -year male vs. 22 year male - at $2 \mathrm{~s}-3 \mathrm{~s}$ & $3.0243 \mathrm{e}+05$ \\
11 -year vs. 22-year male - at $11 \mathrm{~s}-12 \mathrm{~s}$ & $1.5849 \mathrm{e}+05$ \\
11 -year vs. 22-year male - at $21 \mathrm{~s}-22 \mathrm{~s}$ & $3.5597 \mathrm{e}+0$ \\
11 -year male vs. 6-year female - at $2 \mathrm{~s}-3 \mathrm{~s}$ & $6.9878 \mathrm{e}+04$ \\
11 -year male vs. 6-year female - at $11 \mathrm{~s}-12 \mathrm{~s}$ & $7.4862 \mathrm{e}+04$ \\
11 -year male vs. 6-year female - at $21 \mathrm{~s}-22 \mathrm{~s}$ & $3.5022 \mathrm{e}+04$ \\
\hline
\end{tabular}

\section{Conclusions}

Several studies have demonstrated that the left side of the brain is directly connected with logical thinking as well as linguistic functions in the human species (Helmuth et al., 1989; Wackermann, 2004). Also, previous researchers have established that the left side of the brain controls our right side of the body and logical tasks (van Baal et al., 2001; Kreuzer et al., 2014; Corballis, 2014). In our work, we considered raw EEG datasets obtained from the FP1-F7 channels which correspond to the left frontal lobe of the brain. Using the analysis, we were able to produce two conclusions which explain several behaviours related to the subjects. At first, identified behavioural information that more active brain signal patterns occurred from front left area of the brain in young male subjects than in female subjects. Using previous assumptions and with the help of mathematical comparisons, we conclude that young male subjects are more active in logical subjects and linguistic activities than female subjects in the same age group. Subsequently, with the help of cross-correlation and cross-correlation peak value comparisons, we observed high correlation in brain wave patterns between 11-year and 22-year male subjects and also between 11-year male and 6-year female subjects when they are exposed to logical and linguistic tasks. However, it is important to carry out comprehensive clinical trials with subjects in several age groups to make a definite conclusion. 


\section{Acknowledgements}

This work is supported by the University of Sri Jayewardenepura (USJP) under research grant number ASP/01/RE/SCI/2017/10. Experiments were conducted at the Apple Research and Development Centre of the USJP.

\section{References}

Alarcon, G., Guy, C.N. and Binnie, C.D. (2000) 'A simple algorithm for a digital three-pole Butterworth filter of arbitrary cut-off frequency application to digital electroencephalography', Journal of Neuroscience Methods, Vol. 1, No. 1, pp.35-44.

Bickford, R.D., Adelman, G. et al. (1987) 'Electroencephalography', Encyclopaedia of Neuroscience, pp.371-373.

Cantillo-Negrete, J., Gutierrez-Martinez, J., Carino-Escobar, R.I., Carrillo-Mora, P. and Elias-Vinas, D. (2014) 'An approach to improve the performance of subject-independent BCIs-based on motor imagery allocating subjects by gender', Biomedical Engineering Online, Vol. 13, No. 1, p.158.

Corballis, M.C. (2014) 'Left brain, right brain: facts and fantasies', p.e1001767 [online] https://doi.org/10.1371/journal.pbio.1001767.

Goldberger, A.L., Amaral, L.A., Glass, L., Hausdorff, J.M., Ivanov, P.C., Mark, R.G., Mietus, J.E., Moody, G.B., Peng, C.K. and Stanley, H.E. (2000) 'PhysioBank, PhysioToolkit, and PhysioNet: components of a new research resource for complex physiologic signals', Circulation, Vol. 101, No. 23, pp.e215-e220.

Helmuth, S., Fürst, G. and Meyer, B.U. (1989) 'Craniocerebral topography within the international 10-20 system', Electroencephalography and Clinical Neurophysiology, Vol. 72, No. 6, pp.499-506.

Kim, J.W., Lee, Y.S., Han, D.H., Min, K.J., Lee, J. and Lee, K. (2015) 'Diagnostic utility of quantitative EEG in un-medicated schizophrenia', Neuroscience Letters, Vol. 589, pp.126-31.

Kreuzer, I., Osthaus, W.A., Schultz, A. and Schultz, B. (2014) 'Influence of the sevoflurane concentration on the occurrence of epileptiform EEG patterns', PloS One, Vol. 9, No. 2, p.e89191.

Miraglia, F., Vecchio, F., Bramanti, P., Rossini, P.M. et al. (2015) 'Small-worldness characteristics and its gender relation in specific hemispheric networks', Neuroscience, Vol. 310, pp.1-11.

Radin, D.I. (2004) 'Event-related electroencephalographic correlations between isolated human subjects', The Journal of Alternative \& Complementary Medicine, Vol. 10, No. 2, pp.315-323.

Selesnick, I.W., Burrus, S., Mohamad, P.P. et al. (1998) 'Generalized digital Butterworth filter design', IEEE Transactions on Signal Processing, Vol. 46, No. 6, pp.1688-1694.

Tement, A., Pahor, A. and Jaušovec, N. (2016) 'EEG alpha frequency correlates of burnout and depression: the role of gender', Biological Psychology, Vol. 1, No. 2, p.114.

Tsolaki, A., Kosmidou, V., Hadjileontiadis, L., Kompatsiaris, I.Y. and Tsolaki, M. (2015) 'Brain source localization of MMN, P300 and N400: aging and gender differences', Brain Research, Vol. 1603, pp.32-49.

van Baal, G.C.M., Boomsma, D.I., de Geus, E.J.C. et al. (2001) 'Longitudinal genetic analysis of EEG coherence in young wins', Behavior Genetics, Vol. 31, No. 6, pp.637-651.

Wackermann, J. (2004) 'Dyadic correlations between brain functional states: present facts and future perspectives', Mind and Matter, Vol. 2, No. 1, pp.105-122.

Wackermann, J. et al. (2003) 'Correlations between brain electrical activities of two spatially separated human subjects', Neuro-Science letters, Vol. 336, No. 1, pp.60-64.

Zhu, J.Y., Zheng, W.L. and Lu, B.L. (2015) 'Cross-subject and cross-gender emotion classification from EEG', in World Congress on Medical Physics and Biomedical Engineering, Canada, pp.1188-1191. 